\title{
Diffusioosmosis of electrolyte solutions in a fine capillary slit
}

\author{
Hsien Chen Ma, Huan J. Keh* \\ Department of Chemical Engineering, National Taiwan University, Taipei 10617, Taiwan, ROC
}

Received 3 October 2005; accepted 21 November 2005

Available online 20 December 2005

\begin{abstract}
The steady diffusioosmotic flows of an electrolyte solution along a charged plane wall and in a capillary channel between two identical parallel charged plates generated by an imposed tangential concentration gradient are theoretically investigated. The plane walls may have either a constant surface potential or a constant surface charge density. The electrical double layers adjacent to the charged walls may have an arbitrary thickness and their electrostatic potential distributions are determined by the Poisson-Boltzmann equation. Solving a modified Navier-Stokes equation with the constraint of no net electric current arising from the cocurrent diffusion, electric migration, and diffusioosmotic convection of the electrolyte ions, the macroscopic electric field and the fluid velocity along the tangential direction induced by the imposed electrolyte concentration gradient are obtained semianalytically as a function of the lateral position in a self-consistent way. The direction of the diffusioosmotic flow relative to the concentration gradient is determined by the combination of the zeta potential (or surface charge density) of the wall, the properties of the electrolyte solution, and other relevant factors. For a given concentration gradient of an electrolyte along a plane wall, the magnitude of fluid velocity at a position in general increases with an increase in its electrokinetic distance from the wall, but there are exceptions. The effect of the lateral distribution of the induced tangential electric field and the relaxation effect in the double layer on the diffusioosmotic flow are found to be very significant.
\end{abstract}

(C) 2005 Elsevier Inc. All rights reserved.

Keywords: Diffusioosmosis; Plane wall; Capillary slit; Diffusiophoresis

\section{Introduction}

The flow behavior of fluids in porous media is of fundamental and practical interest in various areas of science and engineering. In general, driving forces for the fluid transport through micropores include dynamic pressure differences between the two ends of a capillary pore (convection) [1-6], concentration differences of an impermeable solute between the two bulk solutions outside the pores (osmosis) [4,7], and tangential electrical fields that interact with the electrical double layer adjacent to a charged pore wall (electroosmosis) [2-6,8-13]. Problems of fluid flow induced by these well-known driving forces were treated extensively in the past.

Another driving force for the flow of liquid solutions in a capillary pore, which has commanded less attention, involves concentration gradients of a permeable solute along the cap-

\footnotetext{
* Corresponding author.

E-mail address: huan@ntu.edu.tw (H.J. Keh).
}

illary that interacts with the pore wall. The fluid motion associated with this mechanism is termed diffusioosmosis and has been discussed analytically for solutions of either ionic or nonionic solutes near a plane wall [8,14-19] and inside a capillary pore [20-24]. Some experimental results and interesting applications concerning diffusioosmosis are also available in the literature [25]. In a solution of uncharged solute, the solute molecules interact with the adjacent wall through the van der Waals and dipole forces. For an electrolyte solution in contact with a charged wall, the solute-wall interaction is electrostatic in nature and its range is the Debye screening length $\kappa^{-1}$ (defined right after Eq. (3)). Electrolyte solutions with a concentration gradient of order $100 \mathrm{kmol} / \mathrm{m}^{4}(=1 \mathrm{M} / \mathrm{cm})$ along solid surfaces with a zeta potential of order $k T / e(\sim 25 \mathrm{mV}$; $e$ is the charge of a proton, $k$ is the Boltzmann constant, and $T$ is the absolute temperature) can flow by diffusioosmosis at a velocity of several micrometers per second.

A tangential gradient of a dissociating electrolyte produces fluid flow along a charged solid surface by two mechanisms. The first involves the stresses developed by the tangential gra- 
dient of the excess pressure within the electric double layer (chemiosmotic effect), and the second is based on the macroscopic electric field that is generated because the tangential diffusive and convective fluxes of the two electrolyte ions are not equal (electroosmotic effect). Both mechanisms were considered to some extent in previous investigations for the diffusioosmotic flow [8,14-24]. In these studies, however, either the effect of lateral distributions of the counterions and coions (or of the electrostatic potential) on the local electric field induced by the imposed electrolyte concentration gradient in the tangential direction inside the double layer or the effect of the ionic convection on it caused by the diffusioosmotic flow was neglected.

In this work we present comprehensive analyses of the diffusioosmosis of an electrolyte solution with a constant prescribed concentration gradient along a charged plane wall and in the tangential direction of a capillary channel between two identical parallel charged plates. The zeta potential or surface charge density of the walls is assumed to be uniform, but no assumption is made concerning the magnitude of the electric potential or the thickness of the double layer, and both the lateral distribution of the induced tangential electric field and the effect of the ionic convection on it are allowed. Semianalytical results for the fluid velocity profile and the bulk-phase diffusioosmotic velocity are obtained for various cases. These results show that the effect of the deviation of the induced tangential electric field in the double layer from its bulk-phase quantity and the effect of the ionic convection on the diffusioosmotic velocity of the fluid are dominantly significant in most practical situations, even for the case of a very thin double layer.

\section{Diffusioosmosis of an electrolyte solution along a charged plane wall}

In this section, the diffusioosmotic flow of an infinitely thick solution of a symmetrically charged electrolyte of valence $Z$ (where $Z$ is a positive integer) tangential to a uniformly charged plane wall of length $L$, as illustrated in Fig. 1a, in steady state is

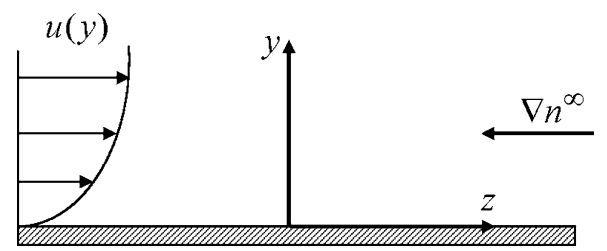

(a)

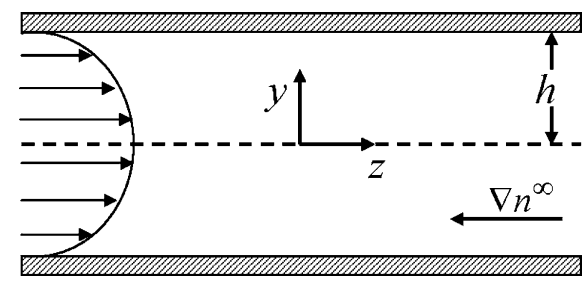

(b)

Fig. 1. Geometrical sketches for the diffusioosmosis due to an applied concentration gradient of an electrolyte: (a) flow tangential to a plane wall; (b) flow in a capillary slit. examined. The applied electrolyte concentration gradient $\nabla n^{\infty}$ is a constant along the tangential $(z)$ direction, where $n^{\infty}(z)$ is the linear concentration (number density) distribution of the electrolyte in the bulk solution phase far from the wall (with $y \rightarrow \infty$ or beyond the electrostatic influence of the charged wall). The end effects are neglected. It is assumed that $n^{\infty}$ is only slightly nonuniform, so that $L\left|\nabla n^{\infty}\right| / n^{\infty}(z=0) \ll 1$, where $z=0$ is set at the midpoint along the plane wall. Thus, the variation of the electrostatic potential and ionic concentrations in the electric double layer adjacent to the wall with the tangential position can be neglected in comparison with their corresponding quantities at $z=0$.

\subsection{Electrostatic potential distribution}

We first consider the electrostatic potential distribution in the electrolyte solution near the charged plane wall. If $\psi(y)$ represents the electrostatic potential at a position $y$ from the wall relative to that in the bulk solution and $n_{+}(y, z)$ and $n_{-}(y, z)$ denote the local concentrations of the cations and anions, respectively, then the Poisson equation gives

$\frac{\mathrm{d}^{2} \psi}{\mathrm{d} y^{2}}=-\frac{4 \pi Z e}{\varepsilon}\left[n_{+}(y, 0)-n_{-}(y, 0)\right]$.

In this equation, $\varepsilon=4 \pi \varepsilon_{0} \varepsilon_{\mathrm{r}}$, where $\varepsilon_{\mathrm{r}}$ is the relative permittivity of the electrolyte solution and $\varepsilon_{0}$ is the permittivity of a vacuum.

The local ionic concentrations can also be related to the electrostatic potential by the Boltzmann equation,

$n_{ \pm}=n^{\infty} \exp (\mp \bar{\psi})$,

where $\bar{\psi}=Z e \psi / k T$ is the dimensionless potential profile. Substitution of Eq. (2) into Eq. (1) results in the well-known Poisson-Boltzmann equation,

$\frac{\mathrm{d}^{2} \bar{\psi}}{\mathrm{d} y^{2}}=\kappa^{2} \sinh \bar{\psi}$

where $\kappa=\left[8 \pi(Z e)^{2} n^{\infty}(z=0) / \varepsilon k T\right]^{1 / 2}$ is the Debye screening parameter.

For the case of constant surface potential, the boundary conditions for $\psi$ are

$y=0: \quad \psi=\zeta$,

$y \rightarrow \infty: \quad \psi=0$,

where the constant $\zeta$ is the zeta potential at the shear plane of the wall adjacent to the electrolyte solution having a uniform bulk concentration $n^{\infty}(z=0)$. The solution to Eqs. (3) and (4) is the well-known Gouy-Chapman result $[8,9,11]$,

$\bar{\psi}=2 \ln \left[\frac{1+\gamma \exp (-\kappa y)}{1-\gamma \exp (-\kappa y)}\right]$,

where $\gamma=\tanh (Z e \zeta / 4 k T)$.

If the constant surface charge density $\sigma$, instead of the surface potential $\zeta$, is known at the plane wall, the boundary condition specified by Eq. (4a) should be replaced by the Gauss 
condition,

$y=0: \quad \frac{\mathrm{d} \psi}{\mathrm{d} y}=-\frac{4 \pi \sigma}{\varepsilon}$.

The solution for $\psi$ given by Eq. (5) still holds for this condition, with the relation between $\zeta$ and $\sigma$ as

$\zeta=\frac{2 k T}{Z e} \sinh ^{-1}\left(\frac{2 \pi Z e \sigma}{\varepsilon \kappa k T}\right)$.

Equation (7) indicates that $\sigma$ increases with an increase in $\kappa$ for the case of constant surface potential and $\zeta$ decreases with an increase in $\kappa$ for the case of constant surface charge density.

\subsection{Induced electric field distribution}

The ionic concentrations $n_{+}$and $n_{-}$in the fluid undergoing diffusioosmosis along the plane wall are not uniform in both tangential $(z)$ and normal $(y)$ directions, and their gradients in the tangential direction can give rise to a "diffusion current" distribution. To prevent a continuous separation of the counterions and coions, an electric field distribution along the tangential direction arises spontaneously in the electrolyte solution to produce another electric current distribution which exactly balances the diffusion current [15-19]. This induced electric field generates an electroosmotic flow of the fluid parallel to the plane wall, in addition to the chemiosmotic flow caused by the electrolyte gradient directly. Both the chemiosmotic and the electroosmotic flows also generate an electric current distribution by ionic convection (known as the relaxation effect), and alternately, this secondary "convection current" again needs to be balanced by the electric current contributed by the induced electric field.

The total flux of either ionic species can be expressed in the general form

$\mathbf{J}_{ \pm}=-D_{ \pm}\left[\nabla n_{ \pm} \pm \frac{Z e}{k T} n_{ \pm}(\nabla \psi-\mathbf{E})\right]+n_{ \pm} \mathbf{u}$,

where $\mathbf{u}=u(y) \mathbf{e}_{z}$ is the fluid velocity relative to the plane wall in the direction of decreasing electrolyte concentration (i.e., $\mathbf{e}_{z}$ is the unit vector in the direction of $\left.-\nabla n^{\infty}\right), D_{+}$and $D_{-}$are the diffusion coefficients of the cations and anions, respectively, $\mathbf{E}=E(y) \mathbf{e}_{z}$ is the macroscopic electric field induced by the concentration gradient of the electrolyte, and the principle of superposition for the electric potential is used. To have no net electric current arising from the cocurrent diffusion (conduction), electric migration, and diffusioosmotic convection of the cations and anions, one must require that $\mathbf{J}_{+}=\mathbf{J}_{-}=\mathbf{J}$ (obviously, the normal component of $\mathbf{J}$ vanishes and the ionic fluxes induced by $\nabla \psi$ in Eq. (8) are balanced by the normal components of the diffusive ionic fluxes as required by the Boltzmann distribution given by Eq. (2)). Applying this constraint to Eq. (8), we obtain

$$
\begin{aligned}
\mathbf{E}= & \frac{k T}{Z e} \frac{\nabla n^{\infty}}{n^{\infty}(z=0)}\left[\frac{(1+\beta) e^{-\bar{\psi}}-(1-\beta) e^{\bar{\psi}}}{(1+\beta) e^{-\bar{\psi}}+(1-\beta) e^{\bar{\psi}}}\right. \\
& \left.+\frac{\operatorname{Pe} \sinh \bar{\psi}}{(1+\beta) e^{-\bar{\psi}}+(1-\beta) e^{\bar{\psi}}} \frac{u}{U^{*}}\right],
\end{aligned}
$$

where

$$
U^{*}=\frac{\varepsilon\left|\nabla n^{\infty}\right|}{4 \pi \eta n^{\infty}(z=0)}\left(\frac{k T}{Z e}\right)^{2}=\frac{2 k T}{\eta \kappa^{2}}\left|\nabla n^{\infty}\right|,
$$

which is a characteristic value of the diffusioosmotic velocity,

$\beta=\frac{D_{+}-D_{-}}{D_{+}+D_{-}}$,

$\mathrm{Pe}=\frac{4 n^{\infty}(z=0) U^{*}}{\left(D_{+}+D_{-}\right)\left|\nabla n^{\infty}\right|}=\frac{8 n^{\infty}(z=0) k T}{\left(D_{+}+D_{-}\right) \eta \kappa^{2}}$,

and $\eta$ is the fluid viscosity. Evidently, $-1 \leqslant \beta \leqslant 1$, with the upper and lower bounds occurring as $D_{-} / D_{+} \rightarrow 0$ and $\infty$, respectively. Typical values of the physical quantities in Eqs. (9)(12) are $U^{*}=10^{-5} \mathrm{~m} / \mathrm{s}, D_{ \pm}=10^{-9} \mathrm{~m}^{2} / \mathrm{s}, n^{\infty}(z=0) /$ $\left|\nabla n^{\infty}\right|=10^{-4} \mathrm{~m}$, and Pe of order unity.

The induced electric filed $\mathbf{E}$ given by Eq. (9) in a selfconsistent way depends on the local electrostatic potential $\psi$ and fluid velocity $u$. It indicates that $\mathbf{E}$ is collinear with and proportional to the tangentially imposed electrolyte gradient $\nabla n^{\infty}$. If we consider the situation that $\kappa y \rightarrow \infty$, then $\psi \rightarrow 0$ and Eq. (9) for the induced electric field caused by the imposed electrolyte concentration gradient reduces to its bulk-phase quantity,

$\mathbf{E}^{\infty}=\frac{k T}{Z e} \frac{\beta \nabla n^{\infty}}{n^{\infty}(z=0)}$.

For the special case of an uncharged wall $(\zeta=0), \mathbf{E}$ at any location $y$ is also identical to this bulk-phase quantity. Note that $\mathbf{E}^{\infty}$ is linearly proportional to the parameter $\beta$, but $\mathbf{E}(y)$ is not necessarily to vanish if $\beta=0$, as shown in Eq. (9).

\subsection{Fluid velocity distribution}

For the steady diffusioosmotic flow along a plane wall, the momentum balances on the symmetric electrolyte solution, which is taken to be incompressible and Newtonian, in the $y$ and $z$ directions give

$$
\begin{aligned}
& \frac{\partial p}{\partial y}+Z e\left(n_{+}-n_{-}\right) \frac{\mathrm{d} \psi}{\mathrm{d} y}=0, \\
& \eta \frac{\mathrm{d}^{2} u}{\mathrm{~d} y^{2}}=\frac{\partial p}{\partial z}-Z e\left(n_{+}-n_{-}\right) E
\end{aligned}
$$

where $p(y, z)$ is the pressure distribution. The boundary conditions for $u$ at the no-slip wall and at infinity are

$$
\begin{aligned}
& y=0: \quad u=0, \\
& y \rightarrow \infty: \quad \frac{\mathrm{d} u}{\mathrm{~d} y}=0 .
\end{aligned}
$$

After the substitution of Eq. (2) into Eq. (14a) based on the assumption that the equilibrium ionic distributions are not affected by the net electrolyte flux $\mathbf{J}$, which is warranted if $\left|\nabla n^{\infty}\right| / \kappa n^{\infty}(z=0) \ll 1$, the pressure distribution can be determined as

$p=p_{\infty}+2 n^{\infty}(z) k T(\cosh \bar{\psi}-1)$. 
Here, $p_{\infty}$ is the pressure far away from the wall, which is a constant in the absence of the applied pressure gradient, and the electric potential distribution $\psi(y)$ is given by Eq. (5).

Substituting the ionic concentration distributions of Eq. (2) and the pressure profile of Eq. (16) into Eq. (14b) and then performing the integration with respect to $y$ twice subject to the boundary conditions in Eqs. (15), we obtain

$$
\begin{aligned}
\frac{u}{U^{*}}= & \int_{0}^{\kappa y} \int_{\infty}^{\kappa y}[\cosh \bar{\psi}-1 \\
& \left.+\frac{Z e n^{\infty}(z=0)}{k T\left|\nabla n^{\infty}\right|} E \sinh \bar{\psi}\right] \mathrm{d}(\kappa y) \mathrm{d}(\kappa y) .
\end{aligned}
$$

After the substitution of Eq. (17) for $u$ and Eq. (5) for $\bar{\psi}$ into Eq. (9), the induced electric field $E$ can be numerically solved as a function of the dimensionless parameters $\kappa y, Z e \zeta / k T, \beta$, and Pe. With the known results of $\bar{\psi}$ and $E$, the diffusioosmotic velocity distribution of the electrolyte solution as a function of $Z e \zeta / k T, \beta$, and Pe can be determined from Eq. (17) with the numerical integrations. Evidently, $u / U^{*}=0$ everywhere if $\zeta=0$. It is understood that, for a given value of $\kappa y$, the quantity $u / U^{*}$ with specified values $-Z e \zeta / k T$ and $\beta$ is equal to that with the values $Z e \zeta / k T$ and $-\beta$. The bulk-phase diffusioosmotic velocity $u_{\infty}$ of the electrolyte solution at a large distance from the plane wall as a function of the relevant parameters can be obtained from the distribution $u$ by taking $\kappa y \rightarrow \infty$.

In previous studies of the diffusioosmosis of electrolyte solutions along a plane wall $[8,15-18]$, the constant bulk-phase quantity of the induced electric field $\mathbf{E}^{\infty}$ given by Eq. (13), taking $\psi=0$ everywhere, was used in Eq. (14b) and its solution gives

$\frac{u_{\infty}}{U^{*}}=\beta \frac{Z e \zeta}{k T}+4 \ln \cosh \left(\frac{Z e \zeta}{4 k T}\right)$.

In this equation, the electroosmotic contribution represented by the first term on the right-hand side and the chemiosmotic contribution denoted by the second term are decoupled as a result of the assumption of a constant induced electric field.

\subsection{Results and discussion}

The distribution of the macroscopic electric field $E(y)$ induced by a concentration gradient of a symmetric electrolyte prescribed parallel to a plane wall can be numerically determined after the substitution of Eqs. (17) and (5) into Eq. (9). A simple method of numerical calculation is to make an initial guess of the fluid velocity distribution $u_{1}(\kappa y)$ for a given combination of the dimensionless parameters $Z e \zeta / k T$, Pe, and $\beta$, and to obtain the resulting induced electric field $E_{1}(\kappa y)$ from Eq. (9). Then, the next result of the velocity distribution $u_{2}(\kappa y)$ can be determined from the double integral involving $E_{1}(\kappa y)$ in Eq. (17). If the difference between $u_{2}(\kappa y)$ and $u_{1}(\kappa y)$ is beyond the tolerable error, the same procedure will be repeated until an acceptable result of the velocity distribution is obtained.

The induced electric field normalized by its bulk-phase quantity, $Z e E n^{\infty}(z=0) / \beta k T\left|\nabla n^{\infty}\right|$, as a function of the electrokinetic distance $\kappa y$ from the wall is plotted in Fig. 2 for

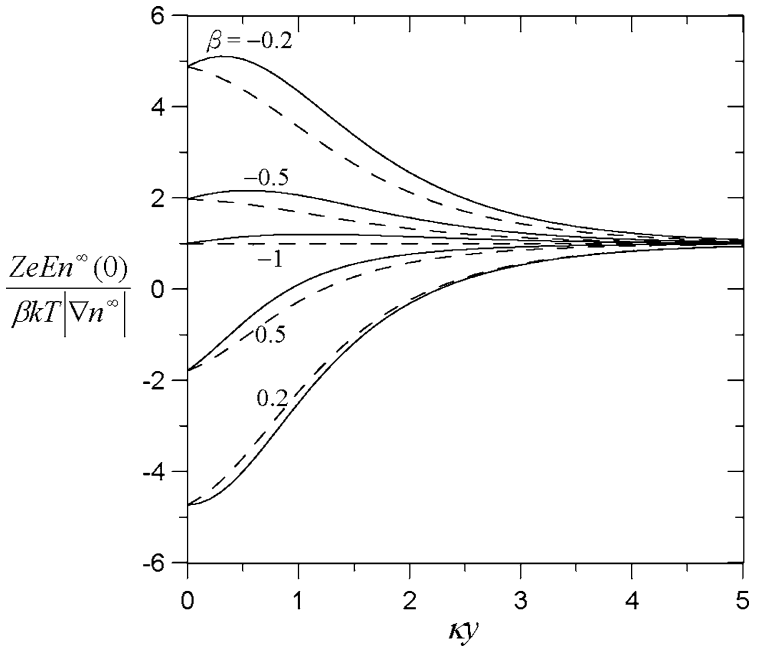

(a)

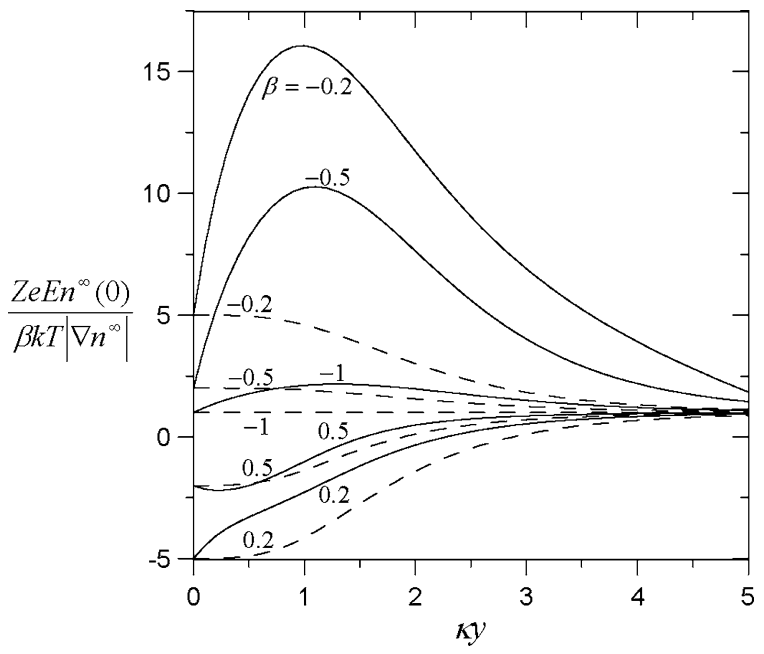

(b)

Fig. 2. Plots of the normalized electric field induced by an electrolyte gradient along a plane wall versus the electrokinetic coordinate $\kappa y$ for various values of the parameter $\beta$ : (a) Ze $\zeta / k T=2$; (b) $Z e \zeta / k T=6$. The solid curves represent the case $\mathrm{Pe}=1$ and the dashed curves denote the case $\mathrm{Pe}=0$.

several values of the parameters $Z e \zeta / k T$, Pe, and $\beta$. Note that each curve with specified values of $-Z e \zeta / k T$ and $\beta$ in this figure would be identical to that with the values $Z e \zeta / k T$ and $-\beta$. As expected, the magnitude of the normalized induced electric field in general is a sensitive function of the electrokinetic coordinate $\kappa y$ and becomes infinity for the special case of $\beta=0$, irrespective of the parameters $Z e \zeta / k T$, $\mathrm{Pe}$, and $\kappa y$. The normalized induced electric field approaches unity (the bulk-phase value) as $\kappa y \geqslant 5$ and has a typical value $-\zeta /|\zeta| \beta$ at the wall (with $\kappa y=0$ ) if $Z e|\zeta| / k T \gg 1$. For the situation that $\zeta \beta>0$, the direction of $\mathbf{E}(y)$ can be opposite to that of its bulk-phase quantity $\mathbf{E}^{\infty}$. When $\mathrm{Pe}=0$, the magnitude of $Z e E n^{\infty}(z=0) / \beta k T\left|\nabla n^{\infty}\right|-1$ (or the deviation of the induced electric field from its bulk-phase quantity) decreases with an increase in $\kappa y$, increases with an increase in $Z e|\zeta| / k T$, and decreases with an increase in the magnitude of $\beta$ (vanishes in the limits of $\beta= \pm 1$ ), for an otherwise specified condition. When the value of Pe is finite, the magnitude of $Z e E n^{\infty}(z=0) / \beta k T\left|\nabla n^{\infty}\right|-1$ may not be a 


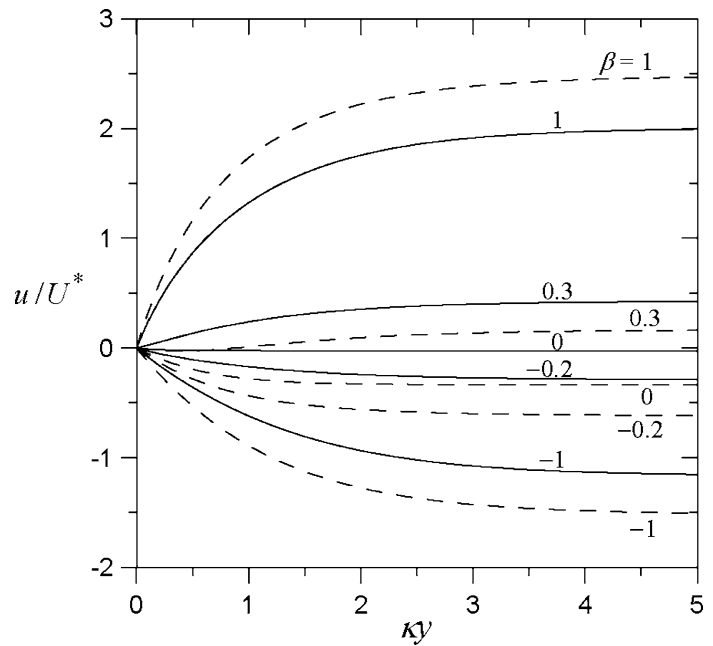

(a)

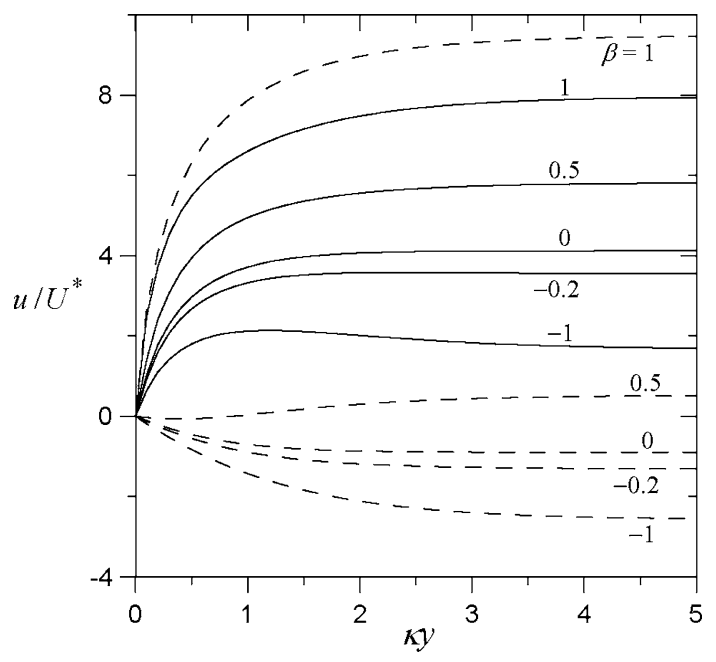

(b)

Fig. 3. Plots of the normalized diffusioosmotic velocity along a plane wall versus the electrokinetic coordinate $\kappa y$ for various values of the parameter $\beta$ : (a) $Z e \zeta / k T=2$; (b) $Z e \zeta / k T=6$. The solid curves represent the case $\mathrm{Pe}=1$ and the dashed curves denote the case $\mathrm{Pe}=0$.

monotonic function of $\kappa y$, and the effect of the electrolyte convection on the local induced electric field in the double layer can be quite significant for the case of high zeta potential at the wall.

The dimensionless diffusioosmotic velocity distribution $u(\kappa y) / U^{*}$ of an electrolyte solution along a plane wall can be numerically calculated using Eq. (17) with the known distributions of $\bar{\psi}$ and $E$. The results are plotted in Figs. 3 and 4 for several values of the parameters $Z e \zeta / k T, \mathrm{Pe}$, and $\beta$. For fixed values of $Z e \zeta / k T, \mathrm{Pe}$, and $\beta$, the value of $u / U^{*}$ approaches a constant (the bulk-phase value $u_{\infty} / U^{*}$ ) as $\kappa y \geqslant 5$. When $\mathrm{Pe}=0$ and $\beta=0$, Eq. (9) indicates that $E$ vanishes everywhere and Fig. 3 shows that the fluid flows toward higher electrolyte concentration ( $u$ is negative) which is due to the chemiosmotic contribution only. When $\mathrm{Pe}=0$ and the product of $\zeta$ and $\beta$ is negative, $u$ is also negative, meaning that the diffusioosmotic flow is in the direction of increasing electrolyte concentration. The magnitude of $u / U^{*}$ increases monotonically with an in-

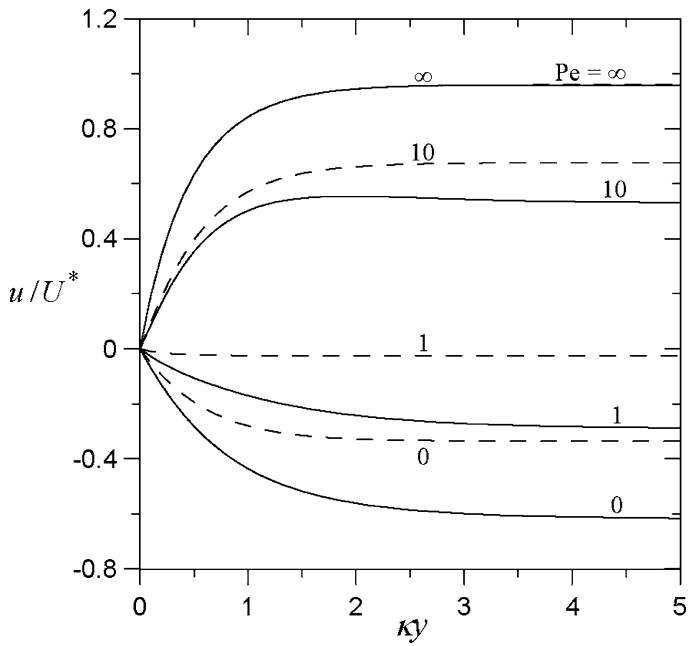

(a)

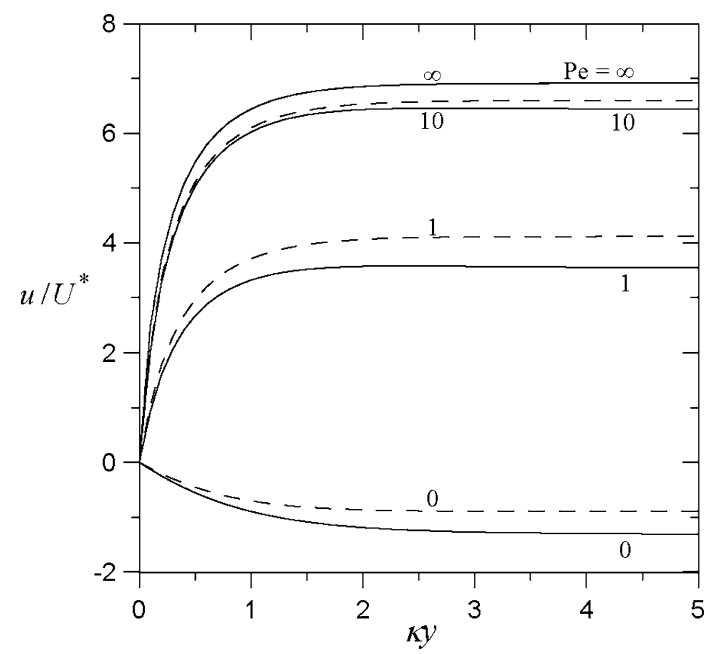

(b)

Fig. 4. Plots of the normalized diffusioosmotic velocity along a plane wall versus the electrokinetic coordinate $\kappa y$ for various values of the parameter Pe: (a) $Z e \zeta / k T=2$; (b) $Z e \zeta / k T=6$. The solid curves represent the case $\beta=-0.2$ and the dashed curves denote the case $\beta=0$.

crease in the electrokinetic distance $\kappa y$ from the wall, with an increase in $Z e|\zeta| / k T$, and with an increase in $|\beta|$, for an otherwise specified condition. When $\mathrm{Pe}=0$ and $\zeta \beta$ is positive, the direction of the diffusioosmotic flow depends on the combination of parameters $Z e \zeta / k T, \beta$, and $\kappa y$. If the magnitude of $\beta$ is sufficiently large, the fluid flows against the electrolyte concentration gradient ( $u$ is positive) and $u / U^{*}$ is a monotonic increasing function of $\kappa y$ and of $Z e|\zeta| / k T$. If the magnitude of $\beta$ is sufficiently small for a given value of $Z e \zeta / k T$, the fluid flows toward the opposite direction ( $u$ is negative) and the magnitude of $u / U^{*}$ is also a monotonic increasing function of $\kappa y$ and of $Z e|\zeta| / k T$. For a specified value of $Z e \zeta / k T$ with an intermediate magnitude of $\beta$, the diffusioosmotic flow may reverse its direction from along with the concentration gradient to against it as the value of $\kappa y$ increases not much from zero.

When the value of Pe is finite, the dependence of $u$ on $\kappa y$ is similar to that for the case of $\mathrm{Pe}=0$ if the value of $Z e|\zeta| / k T$ is small, but $u$ can be positive for any given value of $\beta$ if the 


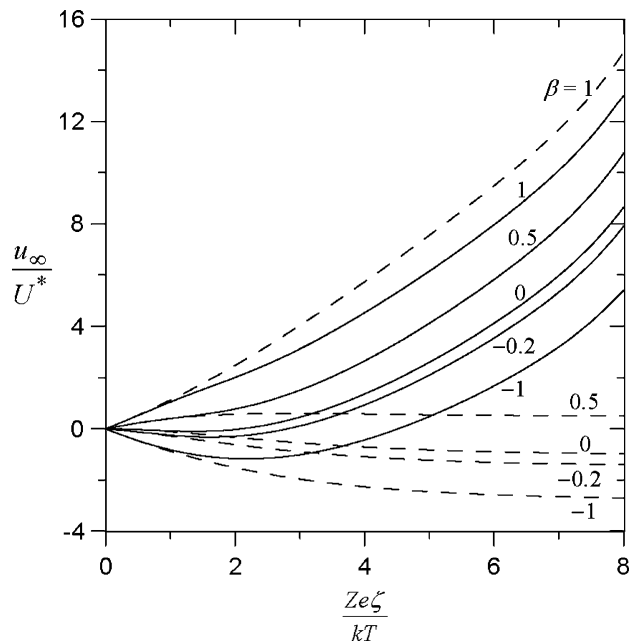

(a)

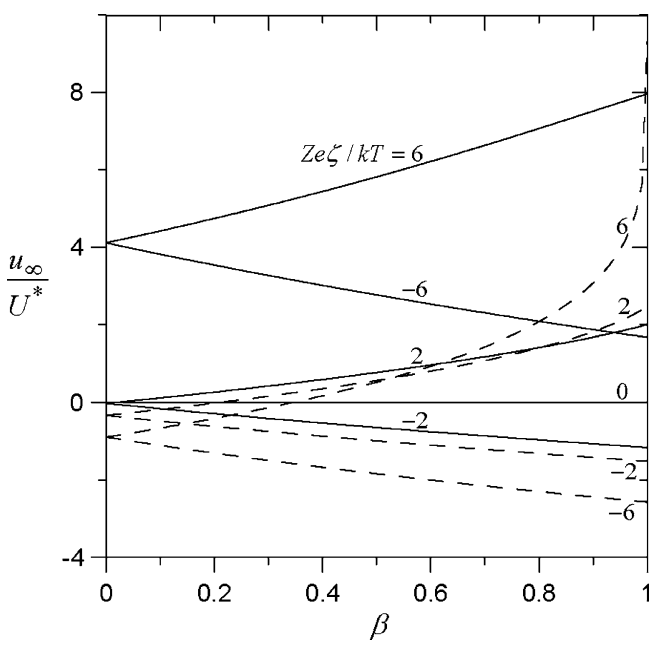

(b)

Fig. 5. The normalized bulk-phase diffusioosmotic velocity along a plane wall: (a) plots versus the dimensionless surface potential $Z e \zeta / k T$ for various values of the parameter $\beta$; (b) plots versus $\beta$ for various values of $Z e \zeta / k T$. The solid curves represent the case $\mathrm{Pe}=1$ and the dashed curves denote the case $\mathrm{Pe}=0$.

value of $Z e|\zeta| / k T$ is large (the relaxation effect on the diffusioosmotic flow is quite significant in this case). In general, the value of $u / U^{*}$ increases monotonically and remarkably with an increase in the value of Pe for specified values of $\kappa y, Z e \zeta / k T$, and $\beta$ except for the situation that the value of $\zeta /|\zeta| \beta$ is close to unity. In the limit of $\mathrm{Pe} \rightarrow \infty, u / U^{*}$ is finite. Note that the cases with $\mathrm{Pe} \geqslant 10$, which are not likely to exist in practice, is exhibited in Fig. 4 for the sake of numerical comparison.

In Fig. 5, the normalized diffusioosmotic velocity $u_{\infty} / U^{*}$ of the electrolyte solution far away from the plane wall is plotted versus the parameters $Z e \zeta / k T$ and $\beta$ for the cases of $\mathrm{Pe}=0$ and 1. Maps showing the direction of this velocity are also

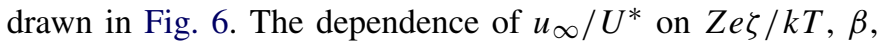
and $\mathrm{Pe}$ is quite similar to that of $u / U^{*}$ for a given value of $\kappa y$. When $\mathrm{Pe}=0$ and the product of $\zeta$ and $\beta$ is negative (in the second and fourth quadrants in Fig. 6a), $u_{\infty}$ is negative and the electrolyte solution flows toward higher concentration. When $\mathrm{Pe}=0$ and the product of $\zeta$ and $\beta$ is positive (in the first and third quadrants in Fig. 6a), the bulk-phase diffusioosmotic ve-

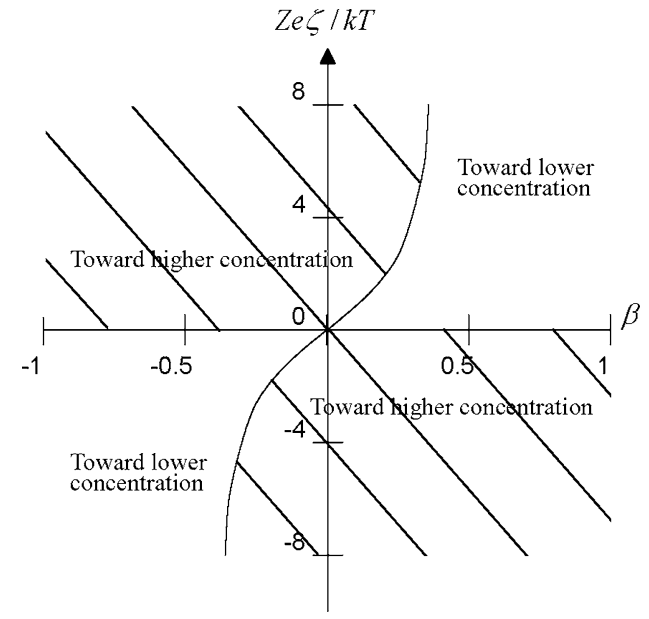

(a)

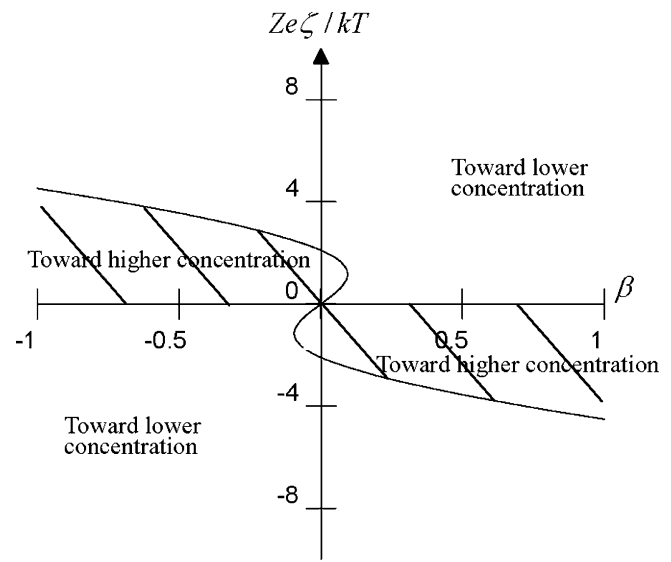

(b)

Fig. 6. Maps showing the direction of the bulk-phase diffusioosmotic velocity along a plane wall: (a) $\mathrm{Pe}=0$; (b) $\mathrm{Pe}=1$.

locity may reverse its direction from against the concentration gradient to along with it as $Z e|\zeta| / k T$ increases not much from zero for the case of a small magnitude of $\beta$ (in addition to a reversal occurring at $\zeta=0$ ), or as $|\beta|$ decreases from 1 to 0 for a given value of $Z e \zeta / k T$. When the value of $\mathrm{Pe}$ is finite, as indicated in Fig. 6b, the probability of the diffusioosmotic flow of the electrolyte solution in the direction toward higher concentration for a combination of $\beta$ and $Z e \zeta / k T$ is greatly reduced, due to the effect of the electrolyte convection. Evidently, this effect is quite significant, irrespective of the thickness of the electric double layer adjacent to the wall.

\section{Diffusioosmosis of an electrolyte solution in a capillary slit}

In this section, we deal with the steady diffusioosmotic flow of a solution of a symmetric electrolyte in a capillary channel between two identical parallel charged plates with a separation distance $2 h$, as shown in Fig. $1 b$. Again, the end effects are neglected. Owing to the planar symmetry of the system, we need consider only the half region $0 \leqslant y \leqslant h$, where $y$ measures distance from the median plane between the capillary walls in a normal direction. The analysis for this case is similar to that 
presented in the previous section for the case of diffusioosmosis along a single plane wall.

\subsection{Electrostatic potential distribution}

For the electrolyte solution in the capillary slit, the electrostatic potential distribution $\psi(y)$ is still governed by Eq. (3), but the boundary conditions become

$y=h: \quad \psi=\zeta$,

$y=0: \quad \frac{\mathrm{d} \psi}{\mathrm{d} y}=0$,

where $\zeta$ is the zeta potential at the capillary walls.

Integration of Eq. (3) from $y=0$ to $y=y$ using Eq. (19b) leads to

$\frac{\mathrm{d} \bar{\psi}}{\mathrm{d} y}=2 \kappa\left[\cosh ^{2}\left(\frac{\bar{\psi}}{2}\right)-\cosh ^{2}\left(\frac{\bar{\psi}_{\mathrm{c}}}{2}\right)\right]^{1 / 2}$,

where $\bar{\psi}_{c}=\bar{\psi}(0)$ represents the potential at the median plane between the slit walls. Integrating Eq. (20) again from $y=0$ to $y=y$, one obtains

$b\left[F\left(b, \frac{\pi}{2}\right)-F(b, \phi)\right]=\kappa y$,

where

$\phi=\sin ^{-1} \frac{\cosh \left(\bar{\psi}_{c} / 2\right)}{\cosh (\bar{\psi} / 2)}$

$b=\left[\cosh \left(\bar{\psi}_{c} / 2\right)\right]^{-1}$,

and $F(b, \phi)$ is the elliptic integral of the first kind,

$F(b, \phi)=\int_{0}^{\phi}\left(1-b^{2} \sin ^{2} \theta\right)^{-1 / 2} \mathrm{~d} \theta$.

Equation (21) is the solution for the potential profile $\psi(y)$ in an implicit form, and the quantity $\psi_{c} / \zeta$ can be determined as a function of the parameters $Z e \zeta / k T$ and $\kappa h$ by Eq. (21) setting $y=h$ and using Eq. (19a).

If the surface charge density $\sigma$, instead of the surface potential $\zeta$, is known at the capillary walls, the boundary condition (19a) should be replaced by the Gauss condition,

$y=h: \quad \frac{\mathrm{d} \psi}{\mathrm{d} y}=\frac{4 \pi \sigma}{\varepsilon}$.

Substitution of this into Eq. (20) yields

$\cosh \frac{Z e \zeta}{k T}-\cosh \frac{Z e \psi_{c}}{k T}=\frac{1}{2}\left(\frac{4 \pi Z e \sigma}{\varepsilon \kappa k T}\right)^{2}$,

which provides the relation between $\zeta$ and $\sigma$ for an arbitrary value of $\kappa h$.

\subsection{Fluid velocity distribution}

The momentum equations for the steady flow of the electrolyte solution in a capillary slit with an applied concentration gradient of the electrolyte parallel to the slit walls are still given by Eqs. (14), and the induced electric field distribution can also be expressed as Eq. (9). The boundary conditions for the fluid velocity $u$ are

$y=h: \quad u=0$,

$y=0: \quad \frac{\mathrm{d} u}{\mathrm{~d} y}=0$.

Substituting Eq. (2) into Eq. (14a), we solve for the pressure distribution to obtain

$p=p_{0}+2 n^{\infty}(z) k T\left(\cosh \bar{\psi}-\cosh \bar{\psi}_{\mathrm{c}}\right)$,

where $p_{0}$ is the pressure on the mid-plane between the slit walls. Substituting Eqs. (28) and (2) into Eq. (14b) and then performing the integration with respect to $y$ twice subject to the boundary conditions in Eqs. (27) yields

$$
\begin{aligned}
\frac{u}{U^{*}}= & \int_{\kappa h}^{\kappa y} \int_{0}^{\kappa y}\left[\cosh \bar{\psi}-\cosh \bar{\psi}_{\mathrm{c}}\right. \\
& \left.+\frac{Z e n^{\infty}(z=0)}{k T\left|\nabla n^{\infty}\right|} E \sinh \bar{\psi}\right] \mathrm{d}(\kappa y) \mathrm{d}(\kappa y) .
\end{aligned}
$$

After the substitution of $u$ expressed by Eq. (29) and $\bar{\psi}$ calculated from Eq. (21) into Eq. (9), the induced electric field $E$ can be numerically solved. With the results of $\bar{\psi}$ and $E$, the diffusioosmotic velocity distribution of the electrolyte solution as a function of the relevant parameters can be calculated from Eq. (29) through the numerical integrations.

\subsection{Results and discussion}

The distribution of the macroscopic electric field $E(y)$ induced by a concentration gradient of a symmetric electrolyte prescribed parallel to the slit walls can be numerically determined after substituting $u / U^{*}$ in the form of Eq. (29) and $\bar{\psi}$ calculated from Eq. (21) into Eq. (9). This induced electric field normalized by its quantity at the midplane between the slit walls, $E(0)$, as a function of the normalized coordinate $y / h$ is plotted in Figs. 7 and 8 for some values of the dimensionless parameters $Z e \zeta / k T, \beta, \mathrm{Pe}$, and $\kappa h$. When $\mathrm{Pe}=0, E(y) / E(0)$ is positive and its value increases with an increase in $y / h$ from unity at the symmetric plane of the slit (with $y=0$ ) to a maximum at the slit wall (with $y=h$ ), increases with an increase in $\kappa h$, decreases with an increase in $Z e|\zeta| / k T$, and increases with an increase in $\zeta /|\zeta| \beta$ if it is not too close to unity (vanishes in the limit of $\zeta /|\zeta| \beta= \pm 1$ ), for an otherwise specified condition. When the value of Pe is finite, the value of $E(y) / E(0)$ is larger than that for the case of $\mathrm{Pe}=0$ if the magnitude of $Z e \zeta / k T$ is small and the value of $\zeta /|\zeta| \beta$ is not too close to unity, but it may not be a monotonic function of $y / h$ and may become negative if the magnitude of $Z e \zeta / k T$ is large. In general, the effect of the electrolyte convection on the local induced electric field in the slit can be quite significant even for the case of low zeta potential at the walls.

The normalized diffusioosmotic velocity distribution $u(y) /$ $U^{*}$ of an electrolyte solution in a slit can be numerically calculated using Eq. (29) with the known results of $\bar{\psi}$ and $E$. This 


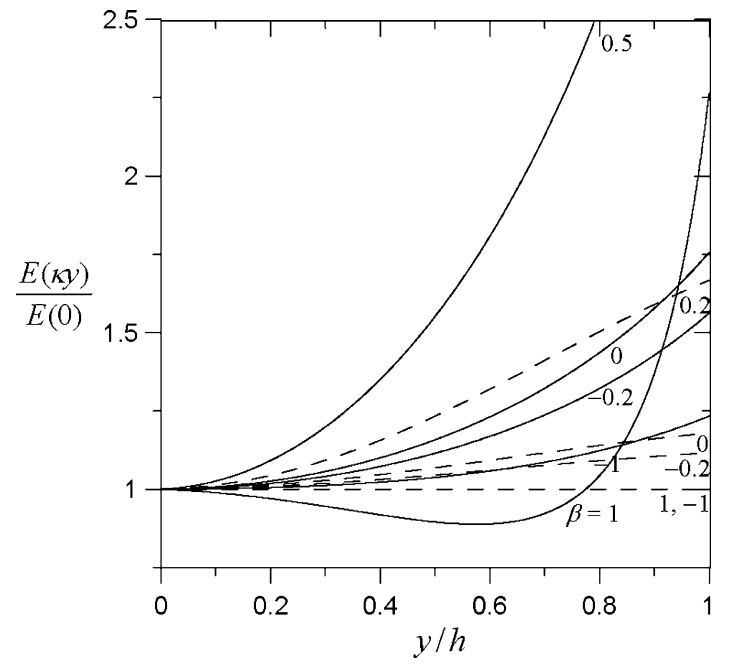

(a)

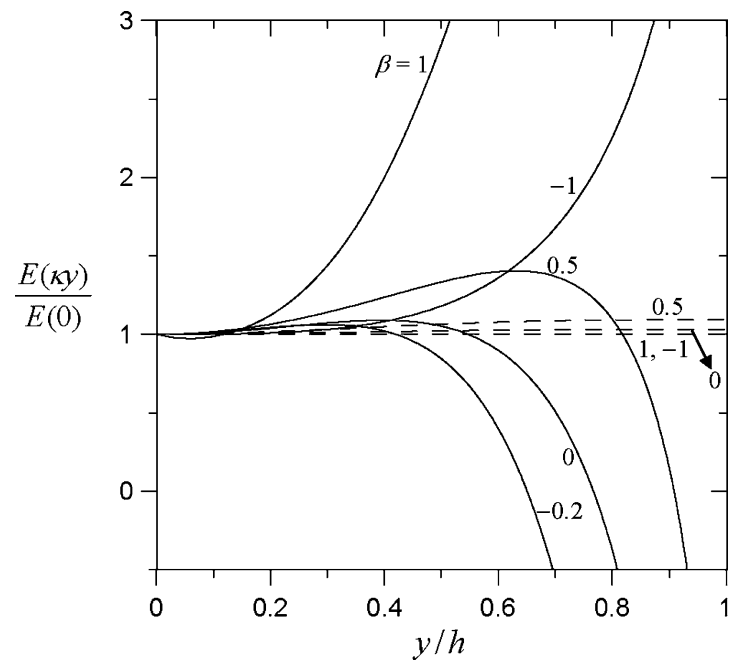

(b)

Fig. 7. Plots of the normalized electric field induced by an electrolyte gradient parallel to the walls of a slit versus the dimensionless coordinate $y / h$ for the case of $\kappa h=1$ with various values of the parameter $\beta$ : (a) $Z e \zeta / k T=2$; (b) $Z e \zeta / k T=6$. The solid curves represent the case $P e=1$ and the dashed curves denote the case $\mathrm{Pe}=0$.

distribution is plotted in Figs. 9 and 10 for several values of the parameters $Z e \zeta / k T, \beta, \mathrm{Pe}$, and $\kappa h$. The diffusioosmotic velocity $u / U^{*}$ is a monotonic increasing function of $\zeta /|\zeta| \beta$. In general, the magnitude of $u / U^{*}$ decreases monotonically with an increase in the normalized coordinate $y / h$. When $\mathrm{Pe}=0$ and $\beta=0$, the fluid flows toward higher electrolyte concentration ( $u$ is negative) which is due to the chemiosmotic contribution only. When $\mathrm{Pe}=0$ and $\zeta /|\zeta| \beta$ is not too close to unity, $u$ is negative, meaning that the diffusioosmotic flow is in the direction of increasing electrolyte concentration. The magnitude of $u / U^{*}$ increases with an increase in $Z e|\zeta| / k T$ and with a decrease in $\zeta /|\zeta| \beta$, for an otherwise specified condition. When $\mathrm{Pe}=0$ and $\zeta /|\zeta| \beta$ approaches unity, the fluid flows against the electrolyte concentration gradient ( $u$ is positive) and $u / U^{*}$ is a monotonic increasing function of $Z e|\zeta| / k T$. When the value of Pe is finite, the dependence of $u$ on $y / h$ is similar to that for

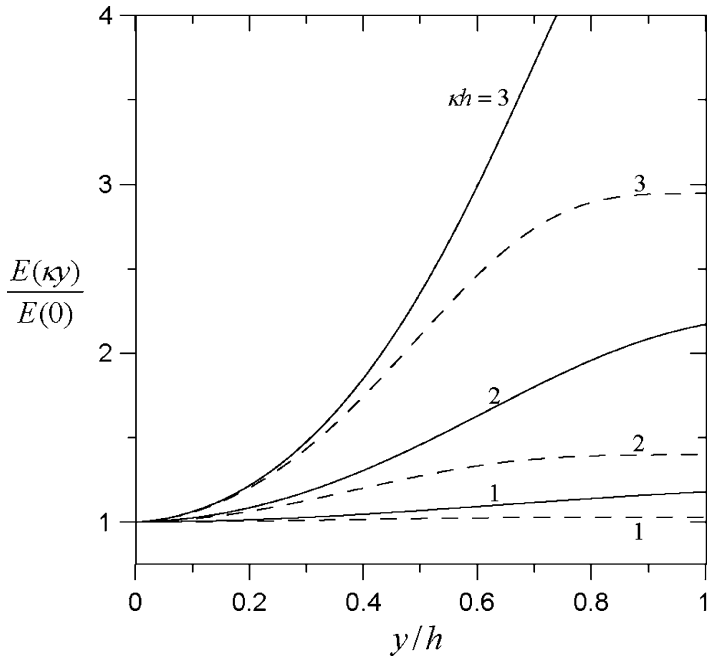

(a)

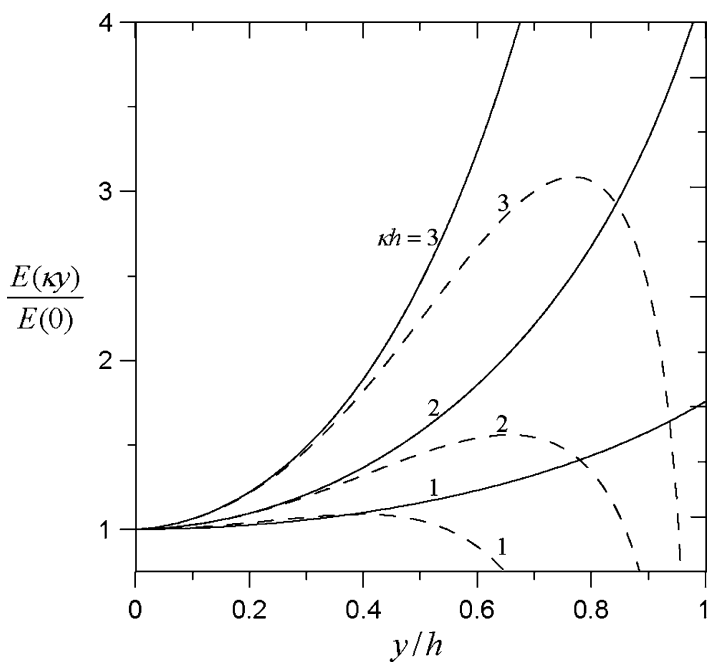

(b)

Fig. 8. Plots of the normalized electric field induced by an electrolyte gradient parallel to the walls of a slit versus the dimensionless coordinate $y / h$ for the case of $\beta=0$ with various values of the parameter $\kappa h$ : (a) $\mathrm{Pe}=0$; (b) $\mathrm{Pe}=1$. The solid curves represent the case $Z e \zeta / k T=2$ and the dashed curves denote the case $Z e \zeta / k T=6$.

the case of $\mathrm{Pe}=0$ if the value of $Z e|\zeta| / k T$ is small, but $u$ can be positive for any given value of $\beta$ if the value of $Z e|\zeta| / k T$ is large. In general, the value of $u / U^{*}$ increases monotonically and strikingly with an increase in the value of $\kappa h$ and with an increase in the value of Pe (the relaxation effect on the diffusioosmotic flow is quite significant) for specified values of $y / h, Z e \zeta / k T$, and $\beta$ except for the situation that the value of $\zeta /|\zeta| \beta$ is close to unity. In the limit of $\mathrm{Pe} \rightarrow \infty, u / U^{*}$ is finite.

In Fig. 11, the normalized diffusioosmotic velocity $u(0) / U^{*}$ of the electrolyte solution at the central plane of the slit is plotted versus the parameters $\kappa h$ and $Z e \zeta / k T$ at specified values of $\mathrm{Pe}$ and $\beta$. Maps showing the direction of this velocity for a typical value of $\kappa h$ are also drawn in Fig. 12. The dependence of $u(0) / U^{*}$ on $Z e \zeta / k T, \beta, \kappa h$, and Pe is quite similar to that of $u / U^{*}$ for a given value of $y / h$. When 


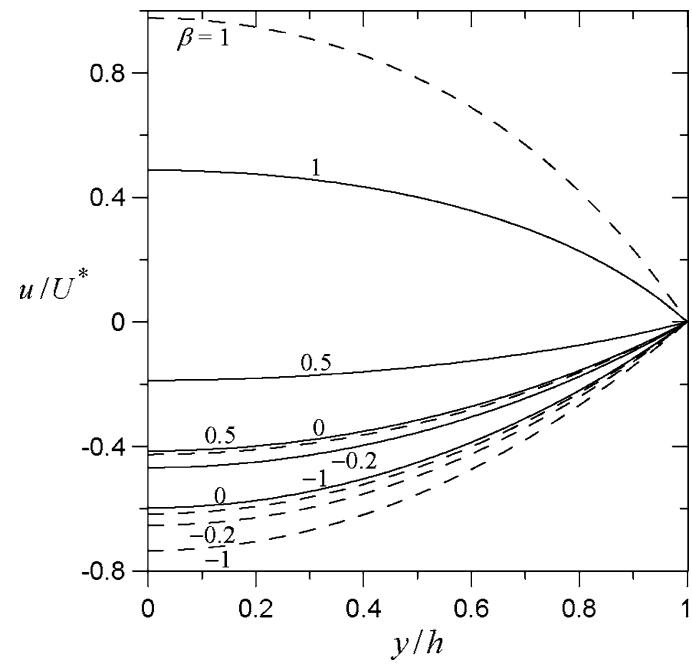

(a)

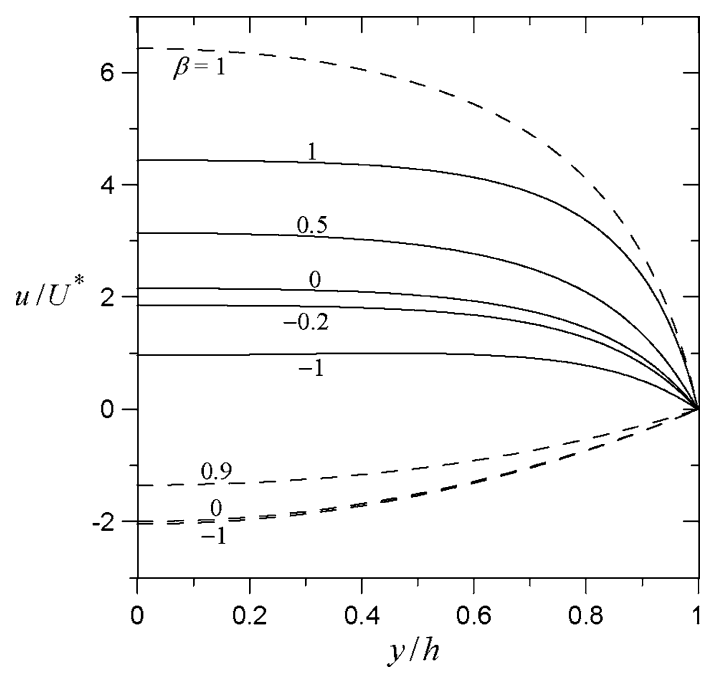

(b)

Fig. 9. Plots of the normalized diffusioosmotic velocity in a slit versus the dimensionless coordinate $y / h$ for the case of $\kappa h=1$ with various values of the parameter $\beta$ : (a) $Z e \zeta / k T=2$; (b) $Z e \zeta / k T=6$. The solid curves represent the case $\mathrm{Pe}=1$ and the dashed curves denote the case $\mathrm{Pe}=0$.

$\mathrm{Pe}=0$ and the product of $\zeta$ and $\beta$ is negative (in the second and fourth quadrants in Fig. 12a), $u_{\infty}$ is negative and the electrolyte solution flows toward higher concentration, analogous to the case of diffusioosmosis along a plane wall discussed in the previous section. When $\mathrm{Pe}=0$ and the product of $\zeta$ and $\beta$ is positive (in the first and third quadrants in Fig. 12a), the bulk-phase diffusioosmotic velocity may reverse its direction from against the concentration gradient to along with it as $Z e|\zeta| / k T$ increases not much from zero for all practical cases of $\beta$ (in addition to a reversal occurring at $\zeta=0$ ), or as $|\beta|$ decreases from 1 to 0 for a small magnitude of $Z e \zeta / k T$. When the value of $\mathrm{Pe}$ is finite, as indicated in Fig. 12b, the probability of the diffusioosmotic flow of the electrolyte solution in the direction toward higher concentration for a combination of $\beta$ and $Z e \zeta / k T$ is again greatly reduced, due to the effect of the electrolyte convection.

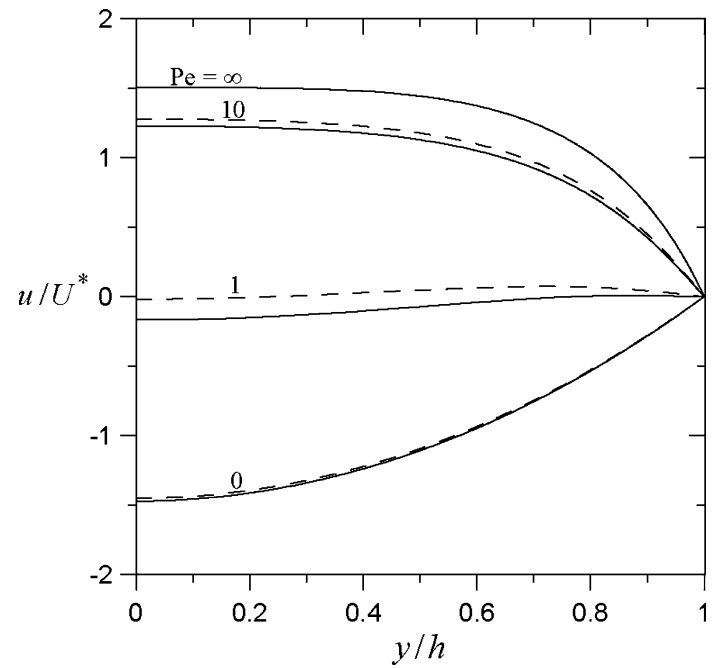

(a)

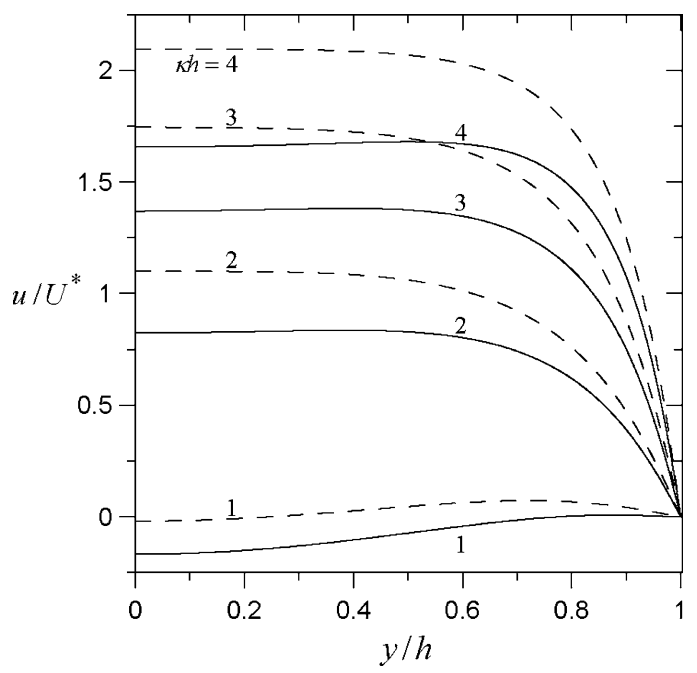

(b)

Fig. 10. Plots of the normalized diffusioosmotic velocity in a slit versus the dimensionless coordinate $y / h$ for the case of $Z e \zeta / k T=4$ : (a) $\kappa h=1$; (b) $\mathrm{Pe}=1$. The solid curves represent the case $\beta=-0.2$ and the dashed curves denote the case $\beta=0$.

\section{Concluding remarks}

A theoretical study of the steady diffusioosmotic flows of solutions of symmetric electrolytes parallel to a charged plane wall and in a capillary slit is presented in this work. It is assumed that the fluid is only slightly nonuniform in the electrolyte concentration along the plane walls, but no assumption is made about the thickness of the electric double layers adjacent to the walls. The effect of lateral distributions of the electrolyte ions (or of the electrostatic potential) and the effect of ionic convection caused by the diffusioosmotic flow (relaxation effect) on the tangential electric field induced by the applied concentration gradient are taken into account. The plane walls may have either a constant surface potential or a constant surface charge density of an arbitrary quantity. By solving the Poisson-Boltzmann equation and the modified Navier-Stokes equation applicable to the system, the electrostatic potential distribution, the induced electric field distribution, and the pres- 


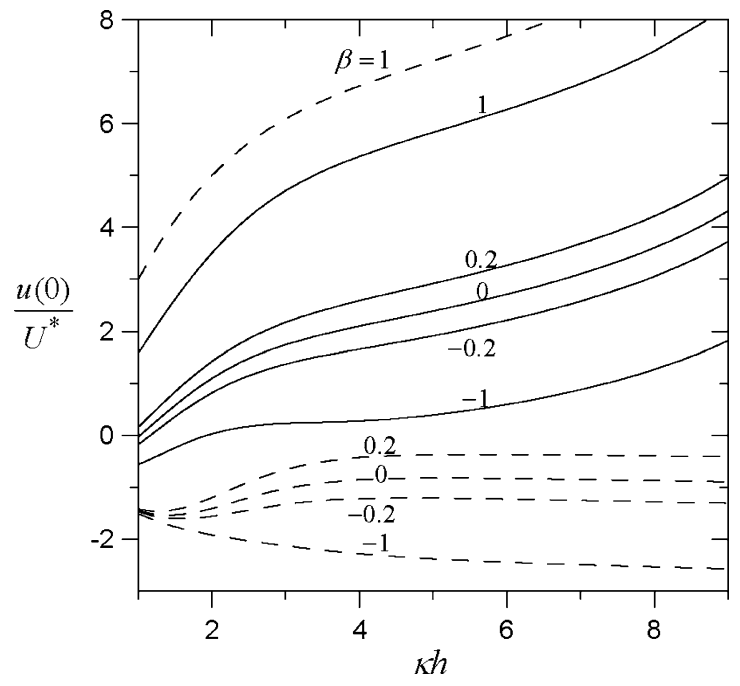

(a)

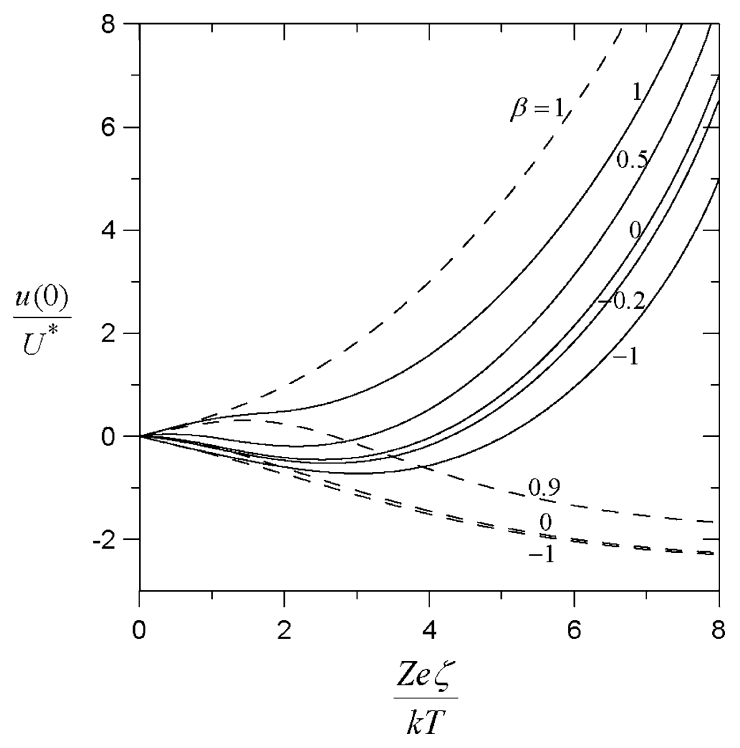

(b)

Fig. 11. The normalized diffusioosmotic velocity at the midplane in a slit for various values of the parameter $\beta$ : (a) plots versus $\kappa h$ for the case of $Z e \zeta / k T=4$; (b) plots versus $Z e \zeta / k T$ for the case of $\kappa h=1$. The solid curves represent the case $\mathrm{Pe}=1$ and the dashed curves denote the case $\mathrm{Pe}=0$.

sure distribution under the influence of the imposed electrolyte gradient are determined either analytically or semianalytically. Numerical results for the local and bulk-phase diffusioosmotic velocities as functions of relevant parameters are presented in detail. The results show that the effect of the deviation of the local induced tangential electric field inside the double layer from its bulk-phase quantity and the relaxation effect can not be neglected in the evaluation of the diffusioosmotic velocity of electrolyte solutions along the walls, even for the case of very thin double layer.

Our analysis also indicates that the velocity variation in the lateral direction for the diffusioosmotic flow of an electrolyte solution can be significant only inside the electric double layer. Therefore, this variation is important for pore size about hundreds of nanometers or smaller as there is an upper limit for the double layer thickness. Some membranes made from thin

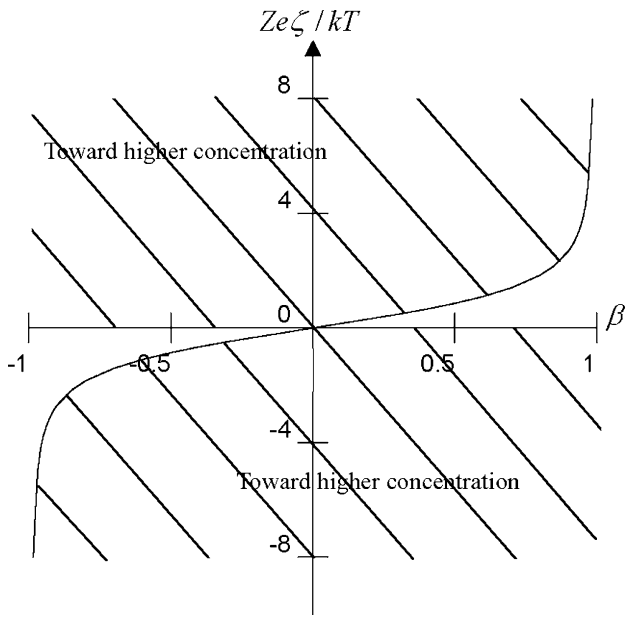

(a)

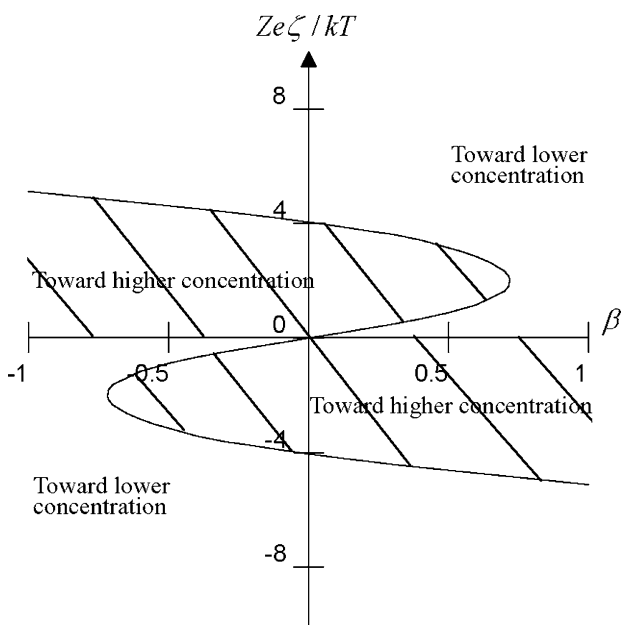

(b)

Fig. 12. Maps showing the direction of the diffusioosmotic velocity at the midplane in a slit for the case of $\kappa h=1$ : (a) $\mathrm{Pe}=0$; (b) $\mathrm{Pe}=1$.

(about $7 \mu \mathrm{m}$ ) sheets of muscovite mica by the track-etch process [22] provide a realistic example with such small pores (the pore radius, defined as the radius of a circle of equivalent area, ranges from several to hundreds nanometers), and a slit might represent such a pore reasonably well (the pores are parallel, cylindrical capillaries of rhombic $\left(60^{\circ}\right)$ cross section).

In contrast to diffusioosmosis, diffusiophoresis refers to the migration of colloidal particles in response to a macroscopic gradient of solute concentration in a solution. Without considering the variation of the induced tangential electric field with the normal position and the effect of ionic convection inside the electric double layer, $u_{\infty}$ given by Eq. (18) has been used as a slip velocity at the surface of a nonconductive charged particle of arbitrary shape with a very thin double layer to derive the steady diffusiophoretic velocity of the particle $[15,16]$. This terminal velocity turned out to be equal in magnitude but opposite in direction to $u_{\infty}$ expressed by Eq. (18). When the effect of the lateral distribution of the induced electric field and the relaxation effect in the double layer are taken into account, the correct diffusiophoretic velocity of the dielectric particle should be equal in magnitude but opposite in direction to the result of $u_{\infty}$ obtained in Section 2 on the basis of the same derivation. 
It is worth repeating that all the results in this study are obtained on the basis of a small external gradient of the electrolyte concentration along the plane wall. If the imposed concentration gradient $\left|\nabla n^{\infty}\right|$ is relatively large, then the effect of variation of the electrostatic potential $\psi$ in the double layer adjacent to the wall with the tangential position may not be neglected. However, it is reasonable for one to expect that this effect will lead to quantitatively rather than qualitatively different results.

\section{Acknowledgment}

This research was supported by the National Science Council of the Republic of China under Grant NSC94-2214-E-002019.

\section{References}

[1] R.B. Bird, W.E. Stewart, E.N. Lightfoot, Transport Phenomena, second ed., Wiley, New York, 2002.

[2] D. Burgreen, F.R. Nakache, J. Phys. Chem. 68 (1964) 1084.

[3] C.L. Rice, R. Whitehead, J. Phys. Chem. 69 (1965) 4017.

[4] A. Szymczyk, B. Aoubiza, P. Fievet, J. Pagetti, J. Colloid Interface Sci. 216 (1999) 285.

[5] H.J. Keh, H.C. Tseng, J. Colloid Interface Sci. 242 (2001) 450.
[6] H.J. Keh, J.M. Ding, J. Colloid Interface Sci. 263 (2003) 645.

[7] J.L. Anderson, D.M. Malone, Biophys. J. 14 (1974) 957.

[8] S.S. Dukhin, B.V. Derjaguin, in: E. Matijevic (Ed.), Surface and Colloid Science, vol. 7, Wiley, New York, 1974.

[9] R.J. Hunter, Zeta Potential in Colloid Science, Academic Press, London, 1981.

[10] H. Ohshima, T. Kondo, J. Colloid Interface Sci. 135 (1990) 443.

[11] J.H. Masliyah, Electrokinetic Transport Phenomena, AOSTRA, Edmonton, 1994.

[12] C. Yang, D. Li, J. Colloid Interface Sci. 194 (1997) 95.

[13] D. Long, H.A. Stone, A. Ajdari, J. Colloid Interface Sci. 212 (1999) 338.

[14] J.L. Anderson, M.E. Lowell, D.C. Prieve, J. Fluid Mech. 117 (1982) 107.

[15] D.C. Prieve, J.L. Anderson, J.P. Ebel, M.E. Lowell, J. Fluid Mech. 148 (1984) 247.

[16] J.L. Anderson, Annu. Rev. Fluid Mech. 21 (1989) 61.

[17] H.J. Keh, S.B. Chen, Langmuir 9 (1993) 1142

[18] Y. Pawar, Y.E. Solomentsev, J.L. Anderson, J. Colloid Interface Sci. 155 (1993) 488.

[19] H.J. Keh, H.C. Ma, Langmuir 21 (2005) 5461.

[20] J.C. Fair, J.F. Osterle, J. Chem. Phys. 54 (1971) 3007.

[21] V. Sasidhar, E. Ruckenstein, J. Colloid Interface Sci. 85 (1982) 332.

[22] G.B. Westermann-Clark, J.L. Anderson, J. Electrochem. Soc. 130 (1983) 839.

[23] H.J. Keh, J.H. Wu, Langmuir 17 (2001) 4216.

[24] H.J. Keh, H.C. Ma, Colloids Surf. A 233 (2004) 87.

[25] S.S. Dukhin, Adv. Colloid Interface Sci. 44 (1993) 1. 